\title{
Three-dimensional magnetic domain analysis by Lorentz Tomography
}

\author{
Akira Yasuhara ${ }^{*}$, Noriaki Endo*, Toshiaki Suzuki ${ }^{*}$ Kazuya Yamazaki ${ }^{*}$, Eiji Okushi ${ }^{*}$, \\ Hiromitsu Furukawa $^{* *}$ and Mike Kersker ${ }^{* * *}$ \\ *JEOL Ltd. 3-1-2 Musashino, Akishima,Tokyo 196-8558, JAPAN \\ **JEOL System Technology Co., Ltd. 3-1-2 Musashino, Akishima,Tokyo 196-8558, \\ JAPAN \\ ***JEOL USA. Inc., 11 Dearborn Rd, Peabody, MA. 01969, U.S.A
}

Recently, TEM tomography is used well in many fields (such as biology, polymer, and catalyst). In this method, a series of step by step tilted TEM images are acquired and a three-dimansional image is reconstructed by a Computerized Tomography (CT). The reconstructed image is analyzed in three-dimensions [1]. We have developed Lorentz tomography. In this new method, a three dimensional image is reconstructed from a series of acquired step by step tilted Lorentz microscope images by the CT method. Magnetic domain structures are analyzed in three-dimensions. By this new method, a structure of a magnetic domain wall and the relationship between a fine structure and a magnetic domain structure can be revealed three-dimensionally. Moreover, we expect that this new method is useful for the analysis of a magnetic characteristic and the development of a new magnetic material.

We show an experimental method and an example of the Lorentz tomography. A series of step by step tilted Lorentz microscope images were acquired with a JEM-2100F. In the case of observing a magnetic domain, the structure was photographed without exciting an objective lens, because a magnetic field of an objective lens tends to destroy an original magnetic domain of a specimen. A specimen was prepared by Bulk Pick-up method with a JEM-9320FIB [2].

A conventional Lorentz microscope image of a SmCo permanent magnet is shown in Fig.1(a) and (b). These images were taken by a Fresnel mode. Bright and dark lines correspond to the position of magnetic domain walls. Three-dimensional reconstructed image of SmCo magnetic domain walls is shown in Fig. 2. Green lines are reconstructed from Fresnel mode images acquired in an under-focus, and White lines are reconstructed from Fresnel mode images acquired in an over-focus. As shown in Fig. 3, magnetic domain walls are observed in the shape of a plane. As a result, the three-dimensional knowledge of a magnetic domain structure can be displayed.

References

[1] H. Furukawa et al, JEOL News, Vol.36 No.1 (2001) 50

[2] T. Suzuki et al, J. Japan Inst. Metals, Vol. 68, No.5 (2004), 293 

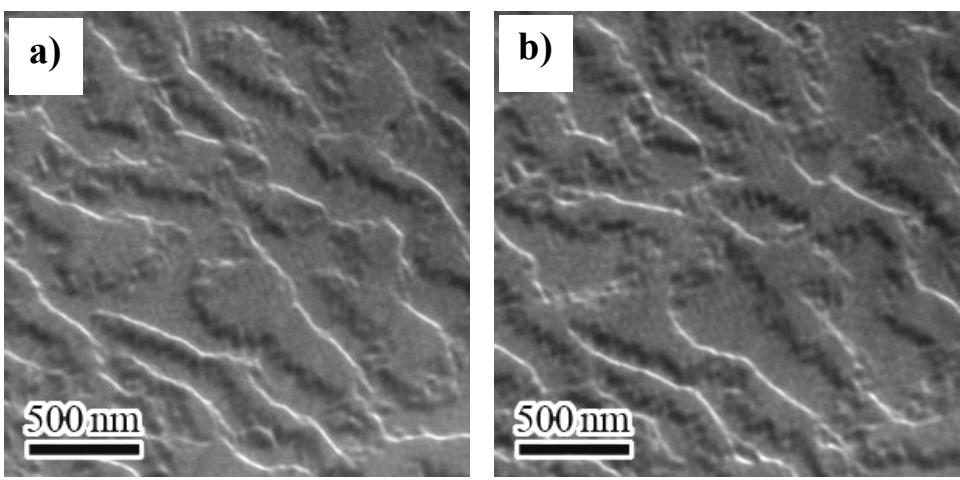

Fig.1. Conventional Lorentz microscope image of a SmCo permanent magnet.

Fresnel mode (a)under-focus image, (b)over-focus image

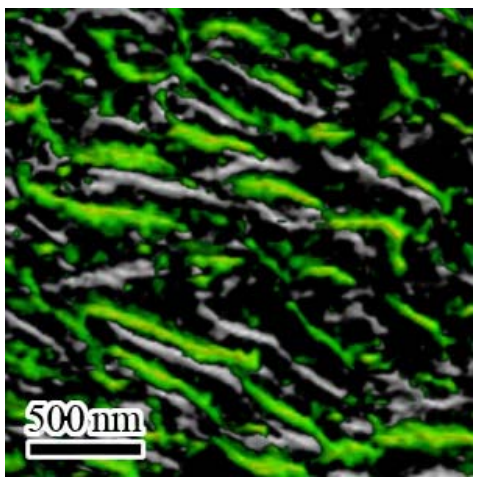

Fig.2. Reconstructed three-dimensional Lorentz microscope image of a SmCo permanent magnet.
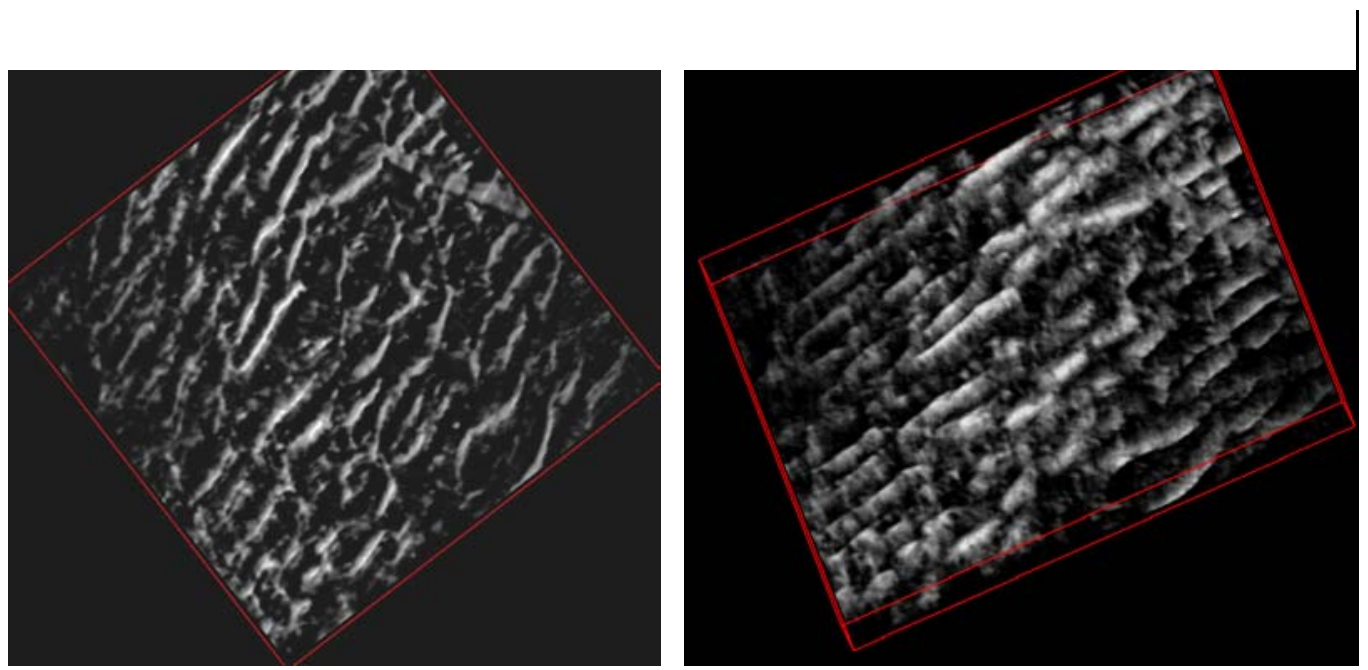

Fig.3. Reconstructed three-dimensional Lorentz microscope image of SmCo permanent magnet (Under-focus image). 